\title{
Ser Hombre, Ser Mujer en la Sexualidad Adolescente
}

\section{Male or Female Sexual Identity in Adolescence}

\author{
Maria Luz Márquez Barradas ${ }^{1}$ \\ Lourdes Pérez Rosiles \\ Beatriz Sibaja Terán
}

\begin{abstract}
Resumen
Los jóvenes expresan un liberalismo sexual que al parecer es más discursivo que práctico; los datos que aquí se presentan muestran que sus actitudes siguen siendo altamente conservadoras. De una muestra de estudiantes de educación media superior se retoman datos sobre su opinión y valoración acerca de algunas cuestiones referidas a la sexualidad, y se presentan en forma comparativa hombres y mujeres con el fin de denotar sus diferencias.
\end{abstract}

Palabras claves: identidad sexual, adolescencia, razón, emoción, conocimiento y practica sexual.

\begin{abstract}
Young people express a sexual liberalism more discursive than practical. This paper shows that their attitudes continue being highly conservative. From a high school sample, data about their opinion and assessmente of some questions referred are compared in order to denote the differences among them.
\end{abstract}

Keywords: Sexual identity, adolescent, reason, emotion, knowledge and sexual practical.

\section{Introducción}

Ser hombre o ser mujer, trasciende la apariencia, incluye el pensar y sentir como tales. Parecer, pensar y sentir como hombre o como mujer son hechos aprendidos, fincados sobre la apariencia física, no en balde en nuestras sociedades el sexo del recién nacido abre la puerta a la expresión de una multiplicidad de comportamientos y expectativas cuya procedencia se verá confirmada en el curso de los años.

La sexualidad es sin duda, uno de los temas que más ocupan la atención de los adul- tos en los análisis de las manifestaciones comportamentales de los jóvenes y por su parte analistas del desarrollo psicosexual proponen que la sexualidad es el eje integrador de la personalidad, es decir, que... "es la sexualidad personal la que hace a cada quien, lo que singulariza para ser entre sus semejantes y/o para confundirse entre la muchedumbre" (COESPO, pag. 29). La sexualidad, integradora de la personalidad y componente esencial en el proceso de identidad social, el que, como bien lo afirma Arriola, (2001) "la identidad constituirá,... un valor negociable", ligado en gran medida al contexto en el

1 Investigadoras del Instituto de Investigaciones Psicológicas, Universidad Veracruzana. Xalapa, Veracruz, México. email:Imarquez@uv.mx 
que los jóvenes - adolescentes- se desenvuelven e influido por factores económicos, sociales y de auto/heteropercepción. En este momento de la vida, los jóvenes parecen actuar en función de sus emociones y su tránsito hacia la adultés, lo es hacia el uso de la razón como eje de su actuar (Di Castro, 1999), y que, por cierto, es el punto hacia donde se encaminan las acciones de los adultos.

Si bien es cierto que los jóvenes adolescentes tienen una serie de comportamientos comunes; el cuestionamiento de la autoridad, la fijación en la congruencia de los adultos, la necesidad de decidir sobre su vida, formas de vestir, de hablar, la inclinación vocacional, también es cierto que ellos y ellas no transitan este momento de la misma manera, y mas aún, su resolución no esta cualitativamente correlacionada entre ellos. Así, decir que los hombres y las mujeres se constituyen de la misma manera puede ser acertado, pero también lo es decir que los contenidos de la identidad-sexualidad, difieren sustancialmente de uno a otra. Porque este periodo de transición reafirma una huella que hace la diferencia entre hombres y mujeres y que en adelante será un rasgo distintivo de identidad; las mujeres dirigidas por su emoción y los hombres con el uso de la razón.

De tal manera que al paso del tiempo, hombres y mujeres llegan a ser los contrapuntos de la emoción y la razón. Este juego de la vida cotidiana, perfila la definición de lo que es ser hombre-ser mujer, de la misma manera que influencia la concepción de ciertos hechos y acciones de la vida como es todo lo referente a la sexualidad. Hay necesidad de hacer una serie de ajustes en la manera de ser y de pensar (Monroy, 1990) para funcionar en una sociedad determinada y consigo mismo, los jóvenes comprenden esta situación, pero también perciben la necesidad de construirse así mismos En ese sentido, la adopción de la regla social como pauta de comportamiento implica, principalmente para los jóvenes, la represión de sus emociones justificada en el uso de la razón.
Pero en la lógica que antes se ha mencionado, el camino hacia la regla social, no es transitado de igual manera entre hombres y mujeres, porque a final de cuentas, como dice Arroyo, "somos en función de nuestras prácticas y del significado colectivo que ellas adquieren .... y por ello, "la infancia, la adolescencia y la vejez son vulnerables a los estereotipos creados en las culturas para excluir y marginar". (Arriola, 2001:16). Así la historia de las mujeres se empieza a escribir con sufrimiento y lagrimas ya que esta mas ligada a la emoción, y la de los hombres con honores y valentía, puesto que su punto fuerte es la razón, por decirlo de una manera coloquial.

Asumir una identidad sexual, comola convicción personal de ser de este o aquel sexo (Craig, 1988), es en todo caso, aprender los papeles propios del sexo-género, adquirir las destrezas y el conocimiento sexual y también -y aquí viene lo más complejo-, entender los comportamientos, formas de pensar y expresarse propios de su rol sexual/género. En este marco general, este trabajo proporciona información sobre las diferencias de género expresadas en las prácticas sexuales, actitudes, intereses y conocimiento sobre salud sexual y reproductiva entre los jóvenes.

Para dar contexto a la información que a continuación se presenta, es necesario considerar que en el Estado de Veracruz, México, habita el $7.08 \%$ de la población total nacional. La población joven (entre 15 y 19 años) en el estado es de 709,056, lo que representa el $10.26 \%$ del total de la población en el mismo, de estos, el $49.23 \%$ son hombres y el $50.77 \%$ mujeres.

El municipio de Xalapa cuenta con 390590 habitantes, de los cuales 39,780 caen en rango de edad mencionada arriba, lo que representa el $5.61 \%$ de la población. De estos, $47.58 \%$ son hombres y $52.15 \%$ son mujeres. La población ubicada en el nivel de preparatoria y/o Bachillerato -nivel escolar que nos ocupa- en el municipio es de 14,861 lo que representa el $37.36 \%$ de la población en el 
rango de edad mencionado, de ellos, $46.15 \%$ son hombres y el $53.85 \%$ son mujeres ${ }^{2}$.

Xalapa es un municipio, que representa el $0.21 \%$ del territorio Veracruzano, su clima es, la mayor parte del año, húmedo y cálido $^{3}$. Designada como capital del estado, es por ello cede de los poderes gubernamentales y municipales, lo cual le da una ambiente de gran dinamismo económico y social. El calificativo de la Atenas Veracruzana le viene de una gran población estudiantil y su ambiente rico en trabajo intelectual.

En este municipio la oferta educativa es variada, hay alrededor de 32 escuelas de nivel medio superior y superior, nivel en que se ubica el Colegio de Bachilleres del Estado de Veracruz (COBAEV) donde se realizo el estudio. Al COBAEV, asisten principalmente jóvenes del municipio lo que caracteriza a su población (de aproximadamente 1200 alumnos en los dos turnos) como urbana y suburbana.

\section{Método y Técnicas}

La escuela donde se llevo a cabo este estudio, trabaja en dos turnos y los programas escolares están organizados por semestres. Así, en el turno matutino cuenta con 16 grupos de alumnos; seis de primer semestre, cinco de tercero y cinco del quinto, de los que se tomó una muestra estratificada.

Se eligieron, al azar, 10 alumnos por grupo, constituyéndose una muestra de 160 alumnos, a los que en colectivo, se les aplicaron, entre otros instrumentos, El Cuestionario de Datos Generales, el Cuestionario de Conocimientos y el de actitudes, cuya información se presenta en este trabajo. Se trata de un estudio de corte transversal, descriptivo cuya finalidad es la detección de problemáticas relativas al ejercicio de la sexualidad en los jóvenes y con la intencionalidad de intervenir.

\section{Resultados}

\section{Información general de los estudiantes}

Los datos generales de los estudiantes: El $65 \%$ vive con su familia, la media de edad es de 16 años; $58 \%$ pertenecen al sexo masculino y $42 \%$ al sexo femenino. En esta población $40 \%$ de los hombres y $16 \%$ de las mujeres tienen vida sexual activa. De estos 35\% de hombres y $15 \%$ de las mujeres reportan el uso de anticonceptivos.

Los chicos inician su vida sexual en un rango que va desde los 10 años, ellas por su parte la inician, en su mayoría, a los 14 años. Sin embargo, quince años parece ser la edad decisiva en los hombres ya que un $41 \%$ de ellos dice haber iniciado su vida sexual a esa edad, a los 14 años solo un 16\% y a los 16 años un $27 \%$ inicio su vida sexual. La mujeres por su parte, muestran una mayor concentración en los 14 años, con un 37\%, le siguen los 15 y 16 años con un $27 \%$ cada uno.

Las siguientes tablas muestran los resultados parciales de sus opiniones y recursos, así, se puede observar que en el caso de las fuentes de información (tabla 1), como fuente unitaria las lecturas tienen un alto porcentaje, y en general las combinaciones entre las opciones de respuesta son importantes.

\section{Tabla 1}

\begin{tabular}{|c|c|c|}
\hline Hombres & & Mujeres \\
\hline $16 \%$ & Maestros & $18 \%$ \\
\hline $15 \%$ & Padres & $5 \%$ \\
\hline $16 \%$ & Amigos & $18 \%$ \\
\hline $20 \%$ & Lecturas Personales & $18 \%$ \\
\hline $2 \%$ & Cine y T. V. & \\
\hline $1 \%$ & No Contestó & $47 \%$ \\
\hline $27 \%$ & Combinaciones & $47 \%$ \\
\hline $3 \%$ & Otros & \\
\hline
\end{tabular}

2 INEGI.(2000) Contar 2000. Sistema para la consulta de tabulados y base de datos de la muestra.

3 INEGI, Gobierno del estado de Veracruz y H. Ayuntamiento de Xalapa. Xalapa. Estado de Veracruz. Cuaderno Estadístico Municipal. Edición 1993. 
La tabla dos muestra la opinión que hombres y mujeres expresan respecto del aborto, donde puede observarse una marcada inclinación hacia el juicio de valor sobre este hecho.

\section{Tabla 2}

\begin{tabular}{|c|c|c|}
\hline Hombres & & Mujeres \\
\hline $13 \%$ & Justificación Médica & $13 \%$ \\
\hline $31 \%$ & Decisión Pareja & $22 \%$ \\
\hline $7 \%$ & Despenalizarse & $8 \%$ \\
\hline $11 \%$ & No Opinan & $18 \%$ \\
\hline $38 \%$ & Homicidio & $36 \%$ \\
\hline & Prob. Salud Pública & $3 \%$ \\
\hline
\end{tabular}

La tabla tres, respecto de las relaciones homosexuales, tanto ellos como ellas se inclinan porque estas sean una toma de decisión libre de cada persona.

Tabla 3

\begin{tabular}{|c|c|c|}
\hline Hombres & & Mujeres \\
\hline $14 \%$ & Inmorales & $15 \%$ \\
\hline $54 \%$ & Libres & $54 \%$ \\
\hline $15 \%$ & Alen. su Prac. & \\
\hline & No Opinan & $18 \%$ \\
\hline $6 \%$ & Práctica Común & $3 \%$ \\
\hline $8 \%$ & Otros & $7 \%$ \\
\hline $3 \%$ & No Contestó & $8 \%$ \\
\hline
\end{tabular}

La tabla cuatro, muestra los resultados al cuestionamiento de porque no han iniciado su vida sexual activa, donde puede observarse que ellas no lo hacen por conservar su virginidad y, en cambio ellos, están esperando que se les presente la oportunidad, en igual medida están preocupados por su virginidad.
Tabla 4

\begin{tabular}{|c|c|c|}
\hline Hombres & & Mujeres \\
\hline $4 \%$ & No Me Atrae & $11 \%$ \\
\hline $20 \%$ & Temor & $9 \%$ \\
\hline $37 \%$ & Oportunidad & $4 \%$ \\
\hline $37 \%$ & Virginidad & $42 \%$ \\
\hline $19 \%$ & Otros & $14 \%$ \\
\hline $14 \%$ & Combinaciones & $20 \%$ \\
\hline
\end{tabular}

La tabla cinco, muestra los motivos esgrimidos para el inicio de su vida sexual activa.

\section{Tabla 5}

\begin{tabular}{|c|c|c|}
\hline Hombres & & Mujeres \\
\hline $16 \%$ & Por Amor & $62 \%$ \\
\hline $21 \%$ & Me lo Pidió mi Pareja & $8 \%$ \\
\hline $18 \%$ & Por Sentir Deseo & $15 \%$ \\
\hline $40 \%$ & Por Experimentar & $0 \%$ \\
\hline $5 \%$ & No Contestó & $15 \%$ \\
\hline
\end{tabular}

La tabla 6 muestra a quien recurrirían en caso de un embarazo no deseado

Tabla 6

\begin{tabular}{|c|c|c|}
\hline Hombres & & Mujeres \\
\hline $59 \%$ & Familiares & $71 \%$ \\
\hline $2 \%$ & Amigos & $2 \%$ \\
\hline $3 \%$ & Evitar & $4 \%$ \\
\hline $12 \%$. & Ayuda Prof & $10 \%$ \\
\hline $10 \%$ & No Se & $3 \%$ \\
\hline $12 \%$ & Otros & $10 \%$ \\
\hline & No Contestó & $3 \%$ \\
\hline
\end{tabular}

La tabla No. 7 muestra los temas de interés para recibir información 
Tabla 7

\begin{tabular}{|c|c|c|}
\hline Hombres & & Mujeres \\
\hline $29 \%$ & Valores y Ética Sexual & $32 \%$ \\
\hline $5 \%$ & Anatomía y Org Sexuales & $9 \%$ \\
\hline $37 \%$ & Act. Sexual H y M & $26 \%$ \\
\hline & Act. Sexual Animal & $3 \%$ \\
\hline $3 \%$ & Concepción Nacimiento & $1 \%$ \\
\hline $9 \%$ & Otros & \\
\hline $2 \%$ & No Contestó & $1 \%$ \\
\hline $15 \%$ & Combinaciones & $28 \%$ \\
\hline
\end{tabular}

\section{Discusión}

En términos generales, la población estudiantil del COBAEV, su ajusta a lo que corresponde a los niveles de educación media superior y superior. La gran mayoría viven con su familia, clase media y media baja y esta es, sin duda, su sostén emocional y económico.

En México, mucho se ha trabajado en cuanto a la educación sexual, proyectos oficiales manejados desde la Secretaría de salud o de Educación, o desde los organismos civiles, han tratado de conformar una cultura sexual cuyos contenidos tienden al cuidado de la salud y donde ellos sean sujetos de credibilidad, los jóvenes participantes en este estudio, sin embargo, expresan una inclinación hacia las fuentes documentales para enterarse sobre estos temas, las chicas por su parte prefieren hablar sobre el tema, no descartan las lecturas, pero al parecer si existe un mayor interés por el trato directo del tema. Es de llamar la atención que los maestros, en ambos casos, juegan un importante papel como informantes, es posible que su estatus social, el tipo de relación, cualitativamente distinta a la de los padres y un cierto ejercicio de poder le asignen una cuota de confiabilidad.

Llama la atención la diferencia en cuanto a confiar en sus padres, los jóvenes los refieren como una fuente reconocida, son también su punto de apoyo latente. No es lo mismo para las chicas, que solo marcan con un 5\%. La experiencia de la sexualidad parece acercar a los jóvenes con sus padres, en cambio a ellas parece alejarlas, lo que puede relacionarse con la represión sexual desigual a la que están sometidos hombres y mujeres. Si un $47 \%$ de las chicas no contesto a esta pregunta, tal vez quiera decir que ese porcentaje esta constituido por las que no hablan con nadie del tema. Y esto es realmente preocupante, si no tienen la práctica del comentario ¿a quien preguntar cuando están en un problema, o simplemente tienen dudas?

Con respecto al aborto, ambos están de acuerdo, en su mayoría, en calificarlo como un homicidio, tal opinión expresa un juicio sumamente conservador y desde luego de carácter moral. Esta opinión es preocupante en términos de que al parecer los jóvenes de hoy no han sido influenciados por los años de lucha a favor de la despenalización, cuyo argumento fundamental es la salud y reconocimiento de los derechos de las mujeres. El hecho es que predomina el juicio moral y que ante esta valoración, una situación de embarazo no planeado puede tener efectos demoledores en su autoestima y autoimagen y marcará de manera definitiva su proyecto de vida. Los jóvenes desarrollan una habilidad que les permite separar su vida personal del resto de la humanidad, y esto podría funcionar como un mecanismo de autodefensa, pero aún así, confrontar su realidad con sus formas de pensar siempre será una experiencia susceptible de generar una situación de conflicto.

En este mismo rublo, un buen porcentaje opina que es una decisión de pareja, es decir, se trata de una situación que ha de resolverse de común acuerdo, donde existen dos involucrados, los que estarían en condiciones de decidir, pero sucede que esto no siempre es posible; cuando ambos son menores de edad y/o dependientes económicos. En todo caso, el apoyo moral y psicológico que pueden darse el uno al otro es digno de tomarse en cuenta. 
Para el caso de la homosexualidad, su opinión es en el sentido de que cada quien es libre de decidir lo que quiera, los hombres pugnan porque de alguna manera se aliente su práctica, por el contrario las mujeres en un buen porcentaje no opinan, y finalmente ambos, con un porcentaje similar las califican de inmorales, coinciden en el juicio moral de los hechos. La opción sexual de compañeros y amigos parece no preocuparlos, en todo caso puede estar expresando un actitud de respeto, el mismo que exigen para si mismos. Por otro lado, puede obedecer a una actitud de "déjennos ser", sin mayor cuestionamiento, en todo caso, su opinión expresa una actitud de mayor tolerancia que las de los adultos, no escapa, sin embargo, al juicio moral de un buen porcentaje, cuya actitud es de intolerancia y crítica, que prejuzga la actuación de los otros.

Las discrepancias en la significación se expresan de igual manera cuando se les cuestiona el porque no tener relaciones sexuales, las mujeres están preocupadas por conservar su virginidad, dato que concuerda con el nacional, lo que muestra una actitud conservadora (Ponce, 2001) con respecto al valor de la virginidad, pese a que el discurso de los adultos sea liberal, y a que los propios adolescentes muestren un comportamiento de tolerancia hacia quienes trasgreden las normas. El temor a las consecuencias es otra de sus razones, y ciertamente ellas, piensan (al menos el 15\%) que el riesgo de un embarazo temprano esta presente, sin embargo, usan menos los anticonceptivos, tal vez -como dice Tolbert (1998)-, las adolescentes prefieren arriesgarse a sufrir las consecuencias de sus actos antes que hacer publico que llevan una sexual activa.

La respuesta de los hombres es concisa, tan solo no han tenido la oportunidad, lo que muestra su actitud valorativa de las relaciones sexuales, son capaces de separar sexo de sentimiento, después de todo el cuerpo tiene sus necesidades (Ardila, 1998), y por lo tanto no hay necesidad de sentirse atraído por una chica, es suficiente con que las condiciones se den, para entrar en esta nueva actividad.

A diferencia de los resultados obtenidos en la Encuesta Nacional de la juventud, 2000, estos jóvenes iniciaron su vida sexual, en promedio, a los 15 años, tres años antes que la media nacional, en el caso que nos ocupa, estos representan el $40 \%$ de la muestra.

La actividad sexual, tiene distintos significados para hombres y mujeres. Mientras las chicas depositan en el sentimiento su decisión de tener relaciones, los hombres las conciben como parte de su experiencia de "conocer el mundo". Es de suponerse que tales significados conllevan construcciones distintas respecto de un mismo hecho, por un lado el romanticismo -cultivar y vivir el sentimiento para si- y por el otro la experimentación -reforzar su hombría y habilidad, para mostrase ante los demás- de ahí que la sexualidad se viva de manera distinta; las mujeres, en su mayoría, sostienen relaciones con sus novios o parejas permanentes y los hombres reportan mas encuentros ocasionales, ellos tienen su espacio para la práctica sexual y ellas, al parecer, son invitadas a distintos lugares.

En caso de un embarazo no deseado, la respuesta es contundente, especialmente en el caso de las mujeres, haciendo mas clara una contradicción -al menos en apariencia-ya que ellas (casi la mitad) no hablan de sexualidad con nadie, pero ante un problema de esta magnitud, su primera intención es acudir a sus familiares Como ya se decía antes, sus padres son su principal fuente de apoyo, pero es predecible la situación de confrontación que esto representa; poca comunicación, una actividad realizada de manera clandestina, padres conservadores, entre otros son los factores que rodean la acción de comunicar a los padres un embarazo prematuro.

Con respecto a los temas de interés, tal vez por las características del cuestionario cuyo contenido incluye aspectos biológicos principalmente, los jóvenes manifiestan un des- 
interés hacia este tema, si bien no expreso, si es indirecto porque eligen otro tipo de temas para se tratados, como lo son, las relaciones hombre/mujer y los valores éticos que les subyacen, es decir se trata de entender la sexualidad. Esto es de suma importancia para programar actividades de atención a su salud sexual, ya que el tema del desarrollo biológico parece agotado. Es interesante hacer notar que este interés pueda estar expresando una preocupación de fondo que tiene que ver con la formación de sus propios valores y principios, los que han regir su manera de comportarse en sociedad, no olvidemos que se trata de adolescentes.

Los resultados muestran que la tendencia a una educación diferenciada existen, que los hombres y las mujeres pueden compartir opiniones, juicios pero en su hacer y ser son diferentes y al mismo tiempo complementarios, la habilidad masculina de separar sus deseos de sus sentimientos parece una constante, en cambio en las mujeres se da, con fuerza el conservadurismo respecto de la virginidad. Valores que finalmente son apoyados, sostenidos y reclamados pos la sociedad, cuya influencia y determinación es innegable.

Para terminar, es necesario visualizar estos resultados a la luz del principio de que los y las adolescentes, están viviendo dos momentos importantes para su futuro; en primer lugar su tránsito de ser niños a ser adultos con sus consecuentes pérdidas y ganancias y en segundo lugar, la modificación de las identidades, que en general, sociedades como la nuestra han generado y cuya base fundamental esta cimentada en las relaciones de género las que, afortunadamente, cada vez tienden mas a la equidad. Esta situación enmarcada en la llamada crisis de identidad, adquiere un significado específico, ya que estaría denotando una condición pasajera, que refiere a la búsqueda de sus intereses y la definición de su personalidad, de ahí la importancia de tomar en cuenta su estado de ánimo, sus intereses y sus capacidades para el diseño y desarrollo de programas de educación de la sexualidad.

\section{Bibliografía}

COESPO. Consejo Nacional de Población. (1982). La educación de la Sexualidad Humana. Colección la educación de la Sexualidad Humana. Educación y Sexualidad. Vol. 4. México.

Ardila, R. (1998) El problema mente cuerpo: implicaciones para la psicología de la salud. Rev. Psicología Contemporánea. Edit. El Manual Moderno. México. 5(2): 4-9

Arriola, A. (2001) Identidad y racismo en este fin de siglo. Edit. FLACSOMAGNATERRA. Guatemala.

D’ Castro, E. (1999). Limites de la subjetividad. Edit Fontamara. México.

Monroy, A; Morales, M. (1990) Salud, Sexualidad y Adolescencia. Edit. Pax México

Craig, G. (1988) Desarrollo psicológico. Prentice-hall Hispanoamericana, S.A. Toronto

Ponce, P., (2001) En sexualidad, nuestra sociedad es profundamente conservadora. Rev. Gaceta de la Universidad Veracruzana. Septiembre 2001. Nueva Epoca. Xalapa. 46: $2-7$

Tolbert, K. (1998). La adolescente embarazada. Características y riesgos psicosociales. Rev. Psicología. Marzo - abril. México. No. 22: 7-13 